\title{
New optical polarization measurements of quasi-stellar objects. The data $\star, \star \star$
}

\author{
D. Sluse ${ }^{1,2}$, D. Hutsemékers ${ }^{1, \star \star \star}$, H. Lamy ${ }^{3}$, R. Cabanac ${ }^{4,5}$, and H. Quintana ${ }^{5}$ \\ ${ }^{1}$ Institut d'Astrophysique et de Géophysique, Université de Liège, Allée du 6 Août 17, B5C, 4000 Liège, Belgium \\ e-mail: sluse@astro.ulg.ac.be \\ 2 European Southern Observatory, Alonso de Cordova 3107, Santiago 19, Chile \\ 3 BIRA - IASB, Avenue Circulaire 3, 1180 Bruxelles, Belgium \\ ${ }^{4}$ Canada France Hawaii Telescope, 65-1238 Mamalahoa Highway, Kamuela, Hawaii 96743, USA \\ 5 Departamento de Astronomía y Astrofísica, Pontificia Universidad Católica de Chile, Casilla 306, Santiago 22, Chile
}

Received 12 October 2004 / Accepted 15 December 2004

\begin{abstract}
New linear polarization measurements (mainly in the $V$ band) are presented for 203 quasi-stellar objects (QSOs). The sample is made up of 94 QSOs located in the North Galactic Pole (NGP) region and of 109 QSOs in the South Galactic Pole (SGP) region. First time measurements have been obtained for 184 QSOs. Among them, 109 known radio-emitters, 42 known Broad Absorption Line (BAL) QSOs, and 1 gravitationally lensed quasi-stellar object. We found high polarization levels $(p>3 \%)$ for 12 QSOs, including the BAL QSO SDSS J1409+0048. For 10 objects, measurements obtained at different epochs do exist. Two of them show evidence for variability: the highly polarized BL Lac candidate PKS 1216-010 and the radio source PKS $1222+037$.
\end{abstract}

Key words. quasars: general - polarization - gravitational lensing

\section{Introduction}

Based on a large sample of quasi-stellar objects with measured optical polarization, Hutsemékers (1998) discovered that there exists regions in the sky where the QSO polarization vectors appear concentrated along preferential directions, on scales up to $\sim 1$ Gpc. New data enabled Hutsemékers \& Lamy (2001) to confirm this effect. In order to obtain an accurate and complete description of this intriguing phenomenon, new QSO polarization measurements are badly needed. The present paper provides a new set of polarimetric data for 94 QSOs located in the North Galactic Cap and for 109 QSOs located in the South Galactic Pole region, with details on the observations, data reduction, and measurements. The comprehensive analysis and the interpretation of the full sample will be reported elsewhere.

This sample may be used for a variety of other studies such as investigating the relation between the optical polarization properties of QSOs and their optical spectra (e.g. Stockman et al. 1984) or their radio properties (e.g. Berriman et al. 1990; Rusk 1990; Visvanathan \& Wills 1998). It also enables one

\footnotetext{
* Based on observations collected at the European Southern Observatory (ESO, La Silla and Paranal).

$\star \star$ Table 4 is only available in electronic form at the CDS via anonymous ftp to cdsarc.u-strasbg.fr $(130.79 .128 .5)$ or via http://cdsweb.u-strasbg.fr/cgi-bin/qcat?J/A+A/433/757 $\star \star \star$ Chercheur qualifié du FNRS, Belgique.
}

to study the polarization properties of sub-classes of QSOs such as Broad Absorption Line (BAL) quasi-stellar objects (e.g. Hutsemékers et al. 1998; Schmidt \& Hines 1999; Lamy \& Hutsemékers 2004) or near-infrared selected QSOs (e.g. Smith et al. 2002).

\section{The observations}

The polarimetric observations were carried out during 5 runs at the European Southern Observatory, La Silla, in August 2000, March 2002, May 2002, August 2003 and October 2003, using the $3.6 \mathrm{~m}$ telescope equipped with EFOSC2. Two additional objects were observed on April 21, 2002 with EFOSC2 and three objects on February 25, 2003 in service mode with the VLT UT1 equipped with the FORS1 camera. The CCD\#40 mounted on EFOSC2 is a $2 \mathrm{k} \times 2 \mathrm{k}$ CCD with a pixel size of $15 \mu \mathrm{m}$ corresponding to $0.158^{\prime \prime}$ on the sky in the $1 \times 1$ binning mode. The standard resolution mode for the $2 \mathrm{k} \times 2 \mathrm{k}$ Tektronix CCD detector of FORS1 has a pixel size of $24 \mu \mathrm{m}$ corresponding to $0.2^{\prime \prime}$ on the sky.

With both the EFOSC2 and FORS1 instruments, linear polarimetry is performed by inserting in the parallel beam a Wollaston prism which splits the incoming light rays into two orthogonally polarized beams. Each object in the field has therefore two orthogonally polarized images on the CCD detector, separated by $20^{\prime \prime}$ for EFOSC 2 and $22^{\prime \prime}$ for FORS 1 . 
To avoid image overlapping, one puts at the telescope focal plane a special mask made of alternating transparent and opaque parallel strips whose width corresponds to the splitting. The object is positioned at the center of a transparent strip which is imaged on a region of the CCD free of defects. The final CCD image then consists of alternate orthogonally polarized strips of the sky, two of them containing the polarized images of the object itself (di Serego Alighieri 1989, 1997; Lamy \& Hutsemékers 1999, hereafter Lam99). Note that the polarization measurements do not depend on variable transparency or seeing since the two orthogonally polarized images of the object are simultaneously recorded.

In order to derive the two normalized Stokes parameters $q$ and $u$ which characterize the linear polarization, frames must be obtained with at least two different orientations of the Wollaston. In practice, the Wollaston is not rotated but a half-wave plate (HWP) is inserted in the optical path and four frames are obtained with the HWP at 4 different position angles $\left(0^{\circ}, 22.5^{\circ}, 45^{\circ}\right.$, and $\left.67.5^{\circ}\right)$. Even if only two different orientations of the HWP are sufficient to retrieve the linear polarization (Melnick et al. 1989), two additional orientations make it possible to remove most of the instrumental polarization (di Serego Alighieri 1989).

Targets were selected from the Véron catalogue (Véron-Cetty \& Véron 2001), from the Sloan Digital Sky Survey Early Data Release (Schneider et al. 2002, 2003; Reichard et al. 2003), from Becker et al. (2000, 2002), Menou et al. (2001), Barkhouse \& Hall (2001), Hall et al. (2002) and Smith et al. (2002), mostly according to their position on the sky i.e. with right ascensions and declinations corresponding to the region of polarization vector alignments defined in Hutsemékers (1998). Bright objects were preferred, as well as BAL, radio-loud and red QSOs which are usually more polarized.

All but two observations were obtained through the Bessel $V$ filter with typical exposure times between 1 and $10 \mathrm{~min}$ per frame. Polarized and unpolarized standard stars were observed in the Bessel $V, R$, and gunn $i$ filters in order to unambiguously fix the zero-point of the polarization position angle and to check the whole observing and reduction process. In August 2000 and October 2003 the sky was clear and the seeing around 1.'0, while in August 2003 conditions were not as good with cirrus and seeing around 1.'5. During the other runs, the seeing was always between $11^{\prime \prime} 1$ and $1{ }^{\prime \prime} 5$, and the sky covered at worst with thin cirrus. A few observations were obtained with a high Moon fraction illumination $(>0.7)$ and are of lower quality. Indeed, high levels of sky background induce larger errors in the sky subtraction process and the subsequent polarization measurements. This is especially relevant when the polarization of the target is low.

\section{Data reduction}

The $q$ and $u$ normalized Stokes parameters are computed from the measurement of the integrated intensity of the orthogonally polarized upper and lower images of the object, for the 4 different orientations of the HWP. They are calculated with respect to the instrumental reference frame using the following formulae:

$$
\begin{aligned}
& q=\frac{R_{q}-1}{R_{q}+1} \quad \text { where } \quad R_{q}^{2}=\frac{I_{0}^{\mathrm{u}} / I_{0}^{1}}{I_{45}^{\mathrm{u}} / I_{45}^{1}} \\
& u=\frac{R_{u}-1}{R_{u}+1} \quad \text { where } \quad R_{u}^{2}=\frac{I_{22.5}^{\mathrm{u}} / I_{22.5}^{1}}{I_{67.5}^{\mathrm{u}} / I_{67.5}^{1}}
\end{aligned}
$$

where $I^{\mathrm{u}}$ and $I^{1}$ respectively refer to the intensities integrated over the upper and lower orthogonally polarized images of the object. The combination of four frames obtained with different HWP orientations not only removes most of the instrumental polarization, but is also essential for correcting the effects of image distortions introduced by the HWP (Lam99).

In order to measure levels of polarization as small as $0.6 \%$ with $0.2 \%$ uncertainty, it is mandatory to achieve photometry with a very high accuracy. For this purpose, the data were first corrected for bias and flat-fielded. The photometric measurements for each image were done using the MIDAS procedure developed by Lam99. The different steps of this procedure are the following: (1) several regions of the background close to the target are interactively chosen; a plane is fitted to their mean values and subtracted from each image individually; (2) the position of the object in each strip is measured at subpixel precision by fitting a 2D Gaussian profile. The flux is subsequently integrated in circles centered at the fitted positions; (3) the Stokes parameters are then computed for any reasonable value of the aperture radius. Since they are found to be stable against radius variation, it was decided to always measure them inside a fixed aperture radius of $3.0 \times\left[(2 \ln 2)^{-1 / 2} H W H M\right]^{1}$ where $H W H M$ represents the mean half-width at half-maximum of the gaussian profile. This empirical choice, seeing independent, is unsensitive to image distorsions (Lam99).

The uncertainties $\sigma_{q}$ and $\sigma_{u}$ on the normalized Stokes $q$ and $u$ are evaluated by computing the errors on the intensities $I^{u}$ and $I^{1}$ from the read-out noise and from the photon noise in the object and the sky background (after converting the counts in electrons), and by propagating these errors. Uncertainties are around $0.15 \%$ for both $q$ and $u$. For a few faint objects listed in Table 1 we were not able to derive reliable measurements, namely due to a higher than usual sky background. These measurements are rejected from the sample presented in Table 4.

A zero-point angle offset correction, filter dependent, is then applied to the QSO normalized Stokes parameters $q$ and $u$ in order to convert the polarization angle measured in the instrumental reference frame into the equatorial reference frame. This angle offset is determined from polarized standard stars observed each night and listed in Table 2. These stars have been selected to have polarization angles distributed in the full $\left[0^{\circ}, 180^{\circ}\right]$ range. For all stars observed during a given run (and between the runs themselves), the values of the angle offset do agree within $1^{\circ}$ standard deviation ${ }^{2}$.

\footnotetext{
${ }^{1}$ For He 1304-1157, the radius was $2.5 \times\left[(2 \ln 2)^{-1 / 2} H W H M\right]$ due to the presence of a cosmic-ray hit at larger radii.

${ }^{2}$ Except the measurements for HD 251204 which disagree in both polarization degree and position angle from tabulated data, possibly indicating polarization variability (see also Weitenbeck 1999).
} 
Table 1. The objects for which no reliable polarization measurements could be derived.

\begin{tabular}{ll}
\hline \hline $\begin{array}{l}\text { Date } \\
\text { dd-mm-yyyy }\end{array}$ & Object \\
\hline $27-08-2000$ & PKS 2357-318 \\
$23-03-2002$ & SDSS J0948+0024, SDSS J1217-0029 \\
$23-03-2002$ & SDSS J1235-0036, PKS 1308+145 \\
$21-04-2002$ & SDSS J1217-0029, SDSS J1235-0036 \\
$02-05-2002$ & CTS A09.82 \\
$20-08-2003$ & PKS 2357-318 \\
$21-08-2003$ & 1WGA J2201.6-5646 \\
$22-08-2003$ & PKS 2314-116 \\
$19-10-2003$ & 4C 19.74 \\
\hline
\end{tabular}

Table 2. The observed standard stars.

\begin{tabular}{llll}
\hline \hline $\begin{array}{l}\text { Date } \\
\text { dd-mm-yyyy }\end{array}$ & Polarized & Unpolarized & Ref. \\
\hline $27-08-2000$ & HD 155197, HD 161056, & HD 154892 & 1 \\
& HD 251204, BD 25 +727 & & 1 \\
$28-08-2000$ & HD 155197, HD 161056 & HD 14069 & 1 \\
$20-03-2002$ & HD 126593, HD 298383 & & 1 \\
$21-03-2002$ & HD 111579, HD 298383 & & 1 \\
$22-03-2002$ & HD 164740, HD 298383 & & 1,2 \\
$23-03-2002$ & HD 111579, HD 155197 & & 1 \\
& HD 298383 & & 1 \\
$21-04-2002$ & HD 111579, HD 155197 & & 1 \\
$01-05-2002$ & HD 155197, HD 298383 & & 1 \\
$02-05-2002$ & HD 111579, HD 298383 & & 3 \\
$25-03-2003$ & Hiltner 652 & & 1 \\
$20-08-2003$ & HD 155197, HD 251204 & HD 154892 & 1 \\
$21-08-2003$ & HD 155197, HD 126593 & & 1 \\
$22-08-2003$ & HD 155197, BD 25 +727 & HD 154892 & 1 \\
$19-10-2003$ & HD 155197, BD 25 +727 & HD 64299 & 1 \\
$19-10-2003$ & HD 155197, HD 298283 & HD 64299 & 1 \\
\hline
\end{tabular}

References: (1) Turnshek et al. (1990); (2) Serkowski et al. (1975); (3) Heiles (2000).

We have also observed several unpolarized standard stars in the $V, R$, and $i$ filters (Table 2). For these stars we measure $p=0.12 \pm 0.05 \%, p=0.08 \pm 0.04 \%$ and $p=0.11 \pm 0.04 \%$ in August 2000, August 2003 and October 2003, respectively, indicating that the residual instrumental polarization is small, a result in agreement with the expectation that most of the instrumental polarization is removed by the observing procedure. No difference between the three filters has been noticed.

Since on most CCD frames field stars are simultaneously recorded, one can use them to estimate the residual instrumental polarization and/or interstellar polarization. While a frameby-frame correction of the QSO Stokes parameters is in principle possible, it is nevertheless hazardous since we are never sure that the polarization of field stars correctly represents the interstellar polarization which could affect distant QSOs. For example, we have found stars located in the same field with significantly different polarization degrees and angles. Also, the field stars are often fainter than the QSO such that
Table 3. The residual polarization.

\begin{tabular}{lrrrr}
\hline \hline $\begin{array}{l}\text { Observing run } \\
\text { mm-yyyy }\end{array}$ & $\begin{array}{r}\bar{q}_{\star} \\
(\%)\end{array}$ & $\begin{array}{c}\bar{u}_{\star} \\
(\%)\end{array}$ & $\begin{array}{c}\bar{\sigma}_{\star} \\
(\%)\end{array}$ & $n_{\star}$ \\
\hline $08-2000$ & +0.04 & -0.01 & 0.19 & 30 \\
$03-2002$ & -0.07 & +0.07 & 0.12 & 57 \\
$05-2002$ & -0.05 & +0.05 & 0.12 & 31 \\
$08-2003$ & -0.10 & -0.18 & 0.24 & 39 \\
$10-2003$ & +0.04 & -0.08 & 0.20 & 31 \\
\hline
\end{tabular}

a frame-by-frame correction would introduce uncertainties on the QSO polarization larger than the correction itself. We then compute the weighted average $\left(\bar{q}_{\star}\right.$ and $\left.\bar{u}_{\star}\right)$ and dispersion $\left(\bar{\sigma}_{\star}\right)$ of the normalized Stokes parameters of field stars considering the $n_{\star}$ frames with suitable stars obtained during a given run. These quantities are given in Table 3 . The small values and dispersions of the residual polarization confirm the small level of uncorrected instrumental polarization. They also indicate that, on average, the interstellar polarization is small, in agreement with the fact that all objects in the sample are at high galactic latitudes $^{3}\left(\left|b_{\mathrm{II}}\right| \geq 30^{\circ}\right)$. To minimize the systematic errors, this residual polarization is conservatively taken into account by subtracting the systematic $\bar{q}_{\star}$ and $\bar{u}_{\star}$ from the measured QSO $q$ and $u$, and by adding quadratically the errors. Since $\bar{q}_{\star}$ and $\bar{u}_{\star}$ are nearly identical in March and May 2002, only the mean values $\bar{q}_{\star}=-0.06 \%, \bar{u}_{\star}=+0.06 \%$ and $\bar{\sigma}_{\star}=0.12 \%$ are used. For those objects observed with FORS1 in February 2003, no correction is done, while the correction used for the March and May 2002 runs is applied to the April 2002 data, obtained with the same instrumental setup.

In order to better understand the nature and the effect of this correction, we illustrate in Fig. 1 (bottom panel) the distribution of the polarization position angles we have measured for field stars ${ }^{4}$ at high galactic latitude $\left(\left|b_{\mathrm{II}}\right| \geq 30^{\circ}\right)$. Polarization data reported in Lamy \& Hutsemékers (2000, hereafter Lam00) and obtained with the same instrumentation are also included. After removing bad quality measurements and redundancies, this leads to a total sample of 204 field star measurements at $\left|b_{\text {II }}\right| \geq 30^{\circ}$, of which about half have polarization angles with $\sigma_{\theta} \leq 14^{\circ}$. The polarization angles of the stars from the Heiles catalogue (Heiles 2000) are illustrated in the top panel of Fig. 1. The distributions are very similar to ours ${ }^{5}$, including definite concentrations of the polarization angles around two main directions: $\sim 70^{\circ}$ towards the NGP and $\sim 135^{\circ}$ towards the SGP. The existence of these two major directions in the interstellar polarization towards the North and the South Galactic Poles has also been reported by Berdyugin et al. (2004), considering distant stars at high galactic latitudes. This similarity in the

\footnotetext{
3 Except FIRST J0809+2753 with $\left|b_{\mathrm{II}}\right|=28.33^{\circ}$.

4 We consider a single star per frame/field. In some cases this star is made up of the combination of several fainter stars from a given frame.

5 There is also a good agreement between the polarization degrees (typically around $0.2-0.3 \%$, cf. Fig. 2), provided that one considers distant stars in the Heiles catalogue, i.e. stars at distances $\geq 100-200$ pc.
} 


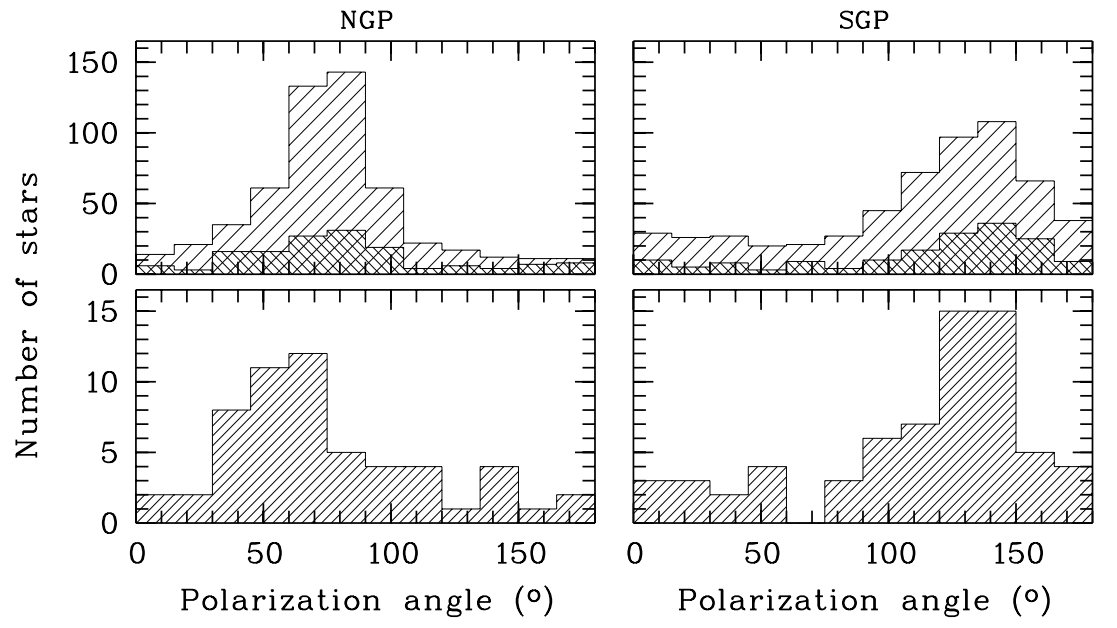

Fig. 1. Top: the distribution of the polarization angles of stars from the Heiles catalogue (Heiles 2000). Only high latitude stars $\left(\left|b_{\mathrm{II}}\right| \geq 30^{\circ}\right)$ with polarization angles having uncertainties $\sigma_{\theta} \leq 14^{\circ}$ are considered. The NGP and SGP regions are illustrated separately. The darker histograms refer to stars at distances $\geq 200$ pc. Bottom: the distribution of the polarization angles of field stars measured on QSO frames. Data from previous papers are included. Only those objects with uncertainties on polarization angles $\sigma_{\theta} \leq 14^{\circ}$ are illustrated.
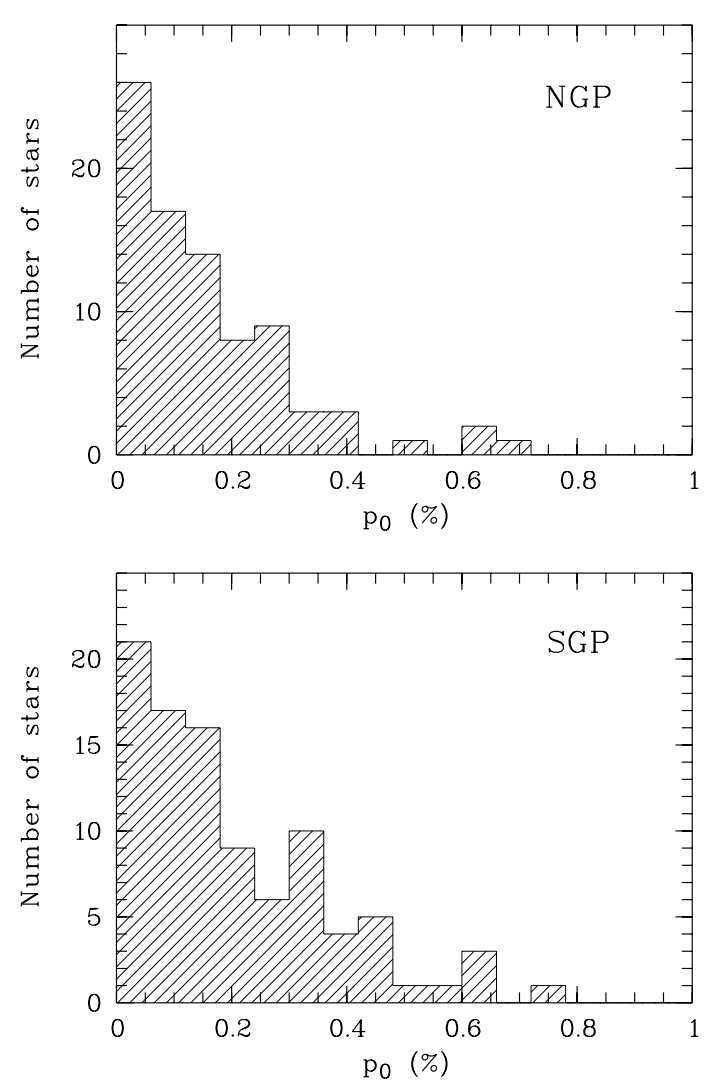

Fig. 2. The distribution of the debiased polarization degree of field stars in the NGP region (top, from the 2002 data) and in the SGP region (bottom, from the 2000 and 2003 data). The correction by the systematic $\bar{q}_{\star}$ and $\bar{u}_{\star}$ is done. Only measurements with an error on the polarization degree $<0.3 \%$ are represented.

polarization angle distributions suggests that a significant part of the polarization we measure for field stars is interstellar in origin. If we average the values of the residual polarization for the NGP and the SGP separately, we get from Table 3 and Lam00, $\bar{q}_{\star}=-0.05 \%$ and $\bar{u}_{\star}=+0.08 \%$ for the NGP, and $\bar{q}_{\star}=-0.01 \%$ and $\bar{u}_{\star}=-0.09 \%$ for the SGP, which correspond to the polarization angles $\bar{\theta}_{\star}=61^{\circ}$ and $\bar{\theta}_{\star}=133^{\circ}$, respectively, in agreement with the trend seen in Fig. 1. Note that a small contribution due to instrumental polarization cannot be excluded given the differences in the values of $\bar{q}_{\star}$ and $\bar{u}_{\star}$ for the various runs (Table 3, Lam00). Although small, the correction by the systematic $\bar{q}_{\star}$ and $\bar{u}_{\star}{ }^{6}$ then removes the bias in the distribution of the polarization angles observed in Fig. 1, at least from the statistical, systematic, point of view.

\section{The results}

Table 4 summarizes the measurements. The first eight columns give the QSO name, type, equatorial coordinates (J2000) and redshift $z$, the date of observation and the normalized Stokes parameters $q$ and $u$ corrected for the systematic residual polarization given in Table 3 . The normalized Stokes parameters are given in the equatorial reference frame. The QSO name is the one used in the Véron catalogue (Véron-Cetty \& Véron 2001) when given, and in the NASA/IPAC Extragalactic Database (NED) otherwise. The name is followed by the object classification. The following notation has been adopted: $R$ if known radio emitter, $B$ if known BAL, $R B$ if both, and $U$ otherwise.

Then, from these values, the polarization degree is evaluated with $p=\left(q^{2}+u^{2}\right)^{1 / 2}$ and the associated error with $\sigma_{p}=\left(\sigma^{2}+\bar{\sigma}_{\star}^{2}\right)^{1 / 2}$ where $\sigma \simeq \sigma_{q} \simeq \sigma_{u}$. In addition, $p$ must be corrected for the statistical bias inherent to the fact that $p$ is always a positive quantity. For this purpose, we used the Wardle \& Kronberg estimator (1974) which was found to be a reasonably good estimator of the true polarization degree (Simmons $\&$ Stewart 1985). The debiased value $p_{0}$ of the polarization degree is reported in Col. 11. The polarization position angle $\theta$ is obtained by solving the equations $q=p \cos 2 \theta$ and $u=p \sin 2 \theta$. The uncertainty of the polarization position angle $\theta$ is estimated from the standard Serkowski (1962) formula where the debiased value $p_{0}$ is conservatively used instead of $p$, i.e. $\sigma_{\theta}=28^{\circ} .65 \sigma_{p} / p_{0}$. Note that due to the HWP chromatism over the $V$ band, an additional error $\leqslant 2-3^{\circ}$ on $\theta$ should be accounted for (cf. the wavelength dependence of the polarization angle offset in di Serego Alighieri 1997).

\footnotetext{
${ }^{6}$ Different $\bar{q}_{\star}$ and $\bar{u}_{\star}$ are used for each observing run. Ideally, as suggested by the results in Fig. 1, one should have also computed $\bar{q}_{\star}$ and $\bar{u}_{\star}$ for $b_{\mathrm{II}} \geq 30^{\circ}$ and $b_{\mathrm{II}} \leq-30^{\circ}$ separately. However this makes little difference since nearly all objects observed in a given run were either at $b_{\mathrm{II}} \geq 30^{\circ}$ or at $b_{\mathrm{II}} \leq-30^{\circ}$.
} 
Figure 2 illustrates the distribution of the field star polarization measured on the QSO frames ${ }^{7}$, for the NGP and the SGP regions separately. For both regions of the sky, the polarization degree is small, most often $\leqslant 0.3 \%$. The distributions are very similar although stars with polarization between $0.3 \%$ and $0.5 \%$ seem slightly more numerous in the SGP. The median polarization is $0.11 \%$ in the NGP region and $0.15 \%$ in the SGP region. The overall distribution suggests that virtually every quasi-stellar object with a polarization higher than $0.6 \%$ is intrinsically polarized, in agreement with previous studies (Berriman et al. 1990; Lam00). In only five cases (flagged in Table 4$)$, the QSO polarization is both significant $(\geqslant 0.6 \%)$ and comparable in degree and angle to the polarization of field stars, suggesting a probable contamination. Four objects with marginal contamination are also indicated in that table.

\subsection{Testing for possible biases in the data}

Since the goal of our polarization measurements is to study concentrations of QSO polarization position angles along preferred directions, it is important to verify that the distribution of polarization angles is not significantly contaminated by either the instrumental polarization or the interstellar polarization in our Galaxy.

\subsubsection{Instrumental polarization}

To verify that the polarization angles are not affected by an instrumental bias, a first test was done using standard stars with intrinsic polarization angles distributed in the full $\left[0^{\circ}, 180^{\circ}\right]$ range (Sect. 2; and Lam00). The excellent agreement - most often within $1^{\circ}$ - between the polarization angles we measure and those values published in the literature demonstrates the absence of such a bias, at least for highly polarized objects.

In order to perform a similar test at lower polarization levels, typically around $1 \%$, we have measured the polarization of a sample of 13 QSOs previously observed by Berriman et al. (1990), Schmidt \& Hines (1999) and Smith et al. (2002) using different telescopes and instruments. The targets were selected to have polarization angles distributed in the full $\left[0^{\circ}, 180^{\circ}\right]$ range (Table 5). Figure 3 shows the observed polarization angle $\theta$ (from Table 4 ) versus the published one $\theta_{l}$ (from Table 5). A good overall agreement is observed, suggesting the absence of an instrumental bias. However, the correlation is not as good as one could have expected given the formal uncertainties. This is due to the fact that we compare unfiltered, white light, measurements to $V$-band polarization angles (with some additional noise from possible time variability). Indeed, while basically constant with wavelength, a slight dependence of the polarization angle on wavelength (i.e. about ten degrees between the blue and red parts of the spectrum) is often observed in low

\footnotetext{
7 We were also able to measure the polarization of 10 field galaxies with a reasonable accuracy. Within the uncertainties, their polarization (both in degree and angle) does not differ from the polarization of the field stars. This suggests that the contamination by interstellar polarization in our Galaxy is not significantly higher for objects at extragalactic distances.
}

Table 5. Published "white light" polarization data for the verification objects.

\begin{tabular}{lllrrl}
\hline \hline Object & $\begin{array}{l}p_{l} \\
(\%)\end{array}$ & $\begin{array}{l}\sigma_{p_{l}} \\
(\%)\end{array}$ & $\begin{array}{r}\theta_{l} \\
\left({ }^{\circ}\right)\end{array}$ & $\begin{array}{r}\sigma_{\theta_{l}} \\
\left({ }^{\circ}\right)\end{array}$ & Ref. \\
\hline PG 0946+301 & 0.85 & 0.14 & 116 & 5 & 1,2 \\
PG 1004+130 & 0.79 & 0.11 & 77 & 4 & 2 \\
PG 1012+008 & 0.66 & 0.23 & 98 & 10 & 2 \\
PKS 1049-09 & 0.85 & 0.30 & 96 & 10 & 2 \\
PG 1216+069 & 0.80 & 0.19 & 53 & 7 & 2 \\
TON 1530 & 0.84 & 0.24 & 150 & 8 & 2 \\
PG 1254+047 & 1.22 & 0.15 & 165 & 3 & 2,1 \\
PG 1435-067 & 1.44 & 0.29 & 27 & 6 & 2 \\
2MA 1519+1838 & 0.67 & 0.22 & 132 & 11 & 3 \\
2MA 1543+1937 & 1.33 & 0.26 & 30 & 6 & 3 \\
3C 323.1 & 1.03 & 0.20 & 4 & 5 & 2 \\
MARK 877 & 0.94 & 0.17 & 79 & 5 & 2 \\
2MA J1714+2602 & 0.86 & 0.33 & 65 & 12 & 3 \\
\hline
\end{tabular}

References: (1) Schmidt \& Hines (1999); (2) Berriman et al. (1990); (3) Smith et al. (2002). When more than one measurement is available (cf. PG 0946+301 and PG 1254+047), the value with the smallest $\sigma_{p_{l}}$ is given.

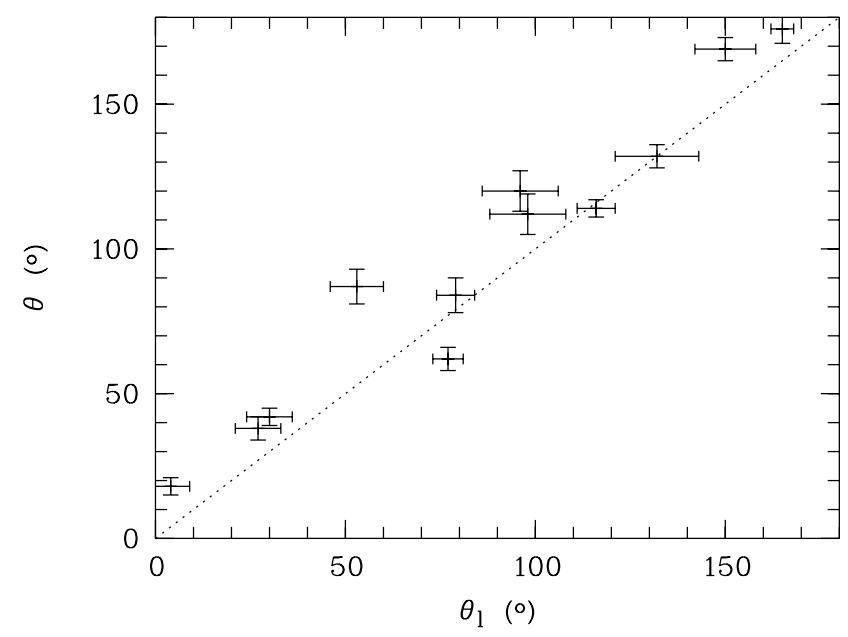

Fig. 3. The measured polarization angle $\theta$ (from Table 4) is reported against the published value $\theta_{l}$ (from Table 5). Due to its very low polarization level and highly uncertain $\theta$ in Table 4, 2MA J1714+2602 is not represented here.

polarization QSOs (Webb et al. 1993; Antonucci et al. 1996). For two objects, $V$-band polarization angle measurements are available in the literature, $\theta=63 \pm 3^{\circ}$ for PG 1004+130 (Webb et al. 1993) and $\theta=23 \pm 2^{\circ}$ for 3C 323.1 (Schmidt \& Smith 2000). They are in much better agreement with our $V$-band measurements than with the white light data. A comparison of polarization measurements obtained in the same filter is then needed to test more accurately for possible instrumental contamination at low polarization levels. 

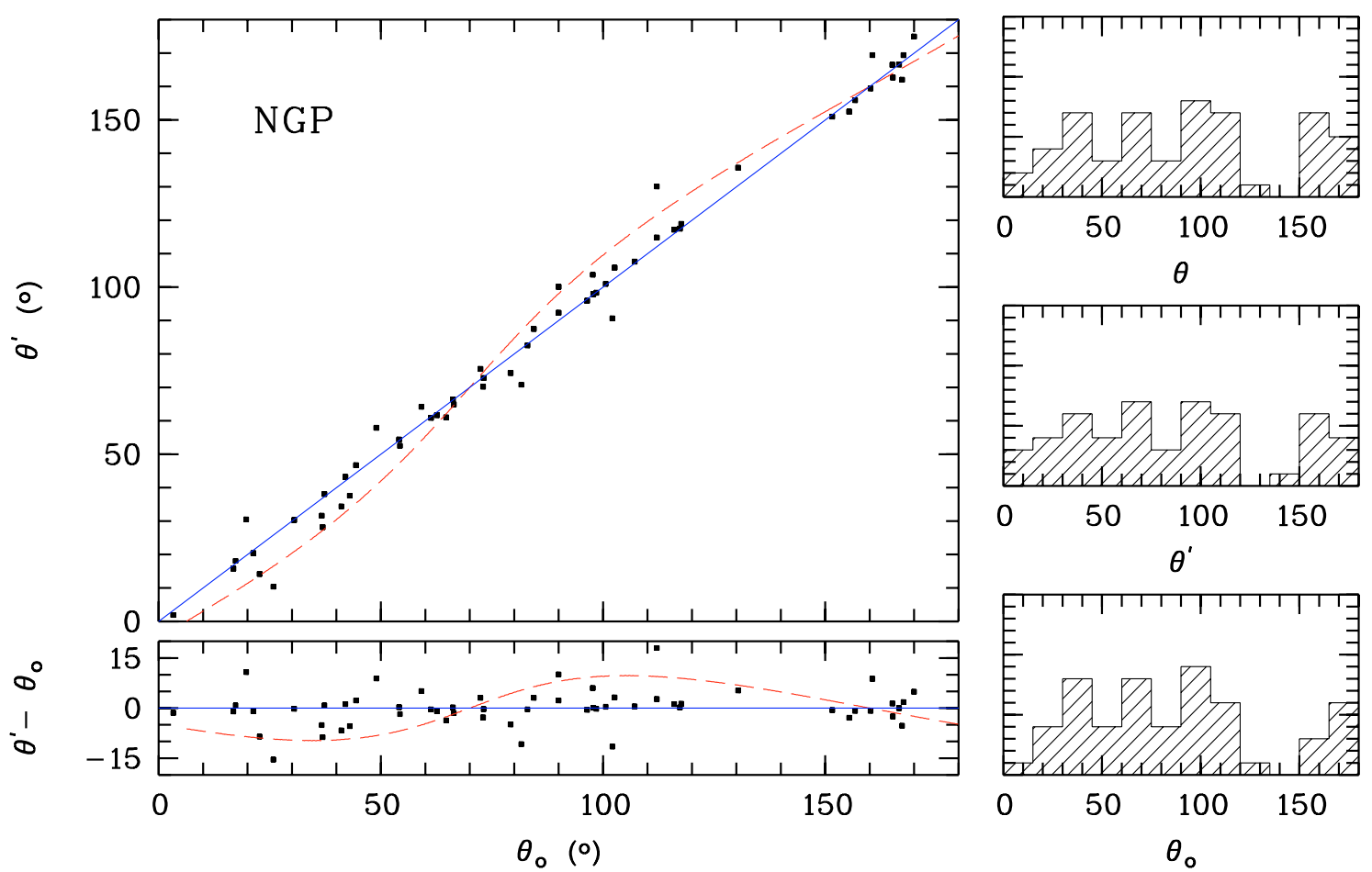

Fig. 4. This figure compares, for the NGP region (2002 data), the QSO polarization angle $\theta_{0}$, uncorrected for the systematic $\bar{q}_{\star}$ and $\bar{u}_{\star}$, to the polarization angle $\theta^{\prime}$ derived after a frame by frame subtraction of the Stokes parameters of field stars (when the latter measurements were possible). Only QSOs with either $p_{0} \geq 0.6 \%$ or $p^{\prime} \geq 0.6 \%$ are plotted. For most objects, $\left|\theta^{\prime}-\theta_{0}\right|$ is within $5^{\circ}$. As a reference, the dashed line represents simulated data obtained by subtracting a systematic polarization of $0.2 \%$ oriented at $70^{\circ}$ (cf. Fig. 1) from a polarization of $0.6 \%$ randomly oriented. Together with the distributions of $\theta_{0}$ and $\theta^{\prime}$, the histograms illustrate the distribution of polarization angle $\theta$ reported in Table 4 and corrected for the systematic $\bar{q}_{\star}$ and $\bar{u}_{\star}$ (QSOs with $p \geq 0.6 \%$ only).

\subsubsection{Interstellar polarization}

In order to estimate the effect of the interstellar polarization on significantly polarized $(p \geq 0.6 \%)$ objects, and namely whether it can introduce a bias in the distribution of the QSO polarization angles, we subtract frame by frame (when possible) the Stokes parameters of the field stars from the QSO ones, both uncorrected for the systematic $\bar{q}_{\star}$ and $\bar{u}_{\star}$. New QSO polarization degrees $\left(p^{\prime}\right)$ and angles $\left(\theta^{\prime}\right)$ are then derived. With such a test, we implicitely assume that the polarization of field stars correctly represents the interstellar polarization affecting the QSOs.

In Figs. 4 and 5 we compare the new polarization angle $\theta^{\prime}$ to the uncorrected one $\theta_{0}$, for the NGP and the SGP regions separately. Only significantly polarized QSOs are considered, i.e. those objects with a polarization degree $\geq 0.6 \%$. The distribution of the final polarization angle $\theta$ given in Table 4 is also illustrated. Although a systematic effect may be noticed, it is small enough to only slightly modify the distribution of the QSO polarization angles. This is due to the fact that the field star polarization is most often small (Fig. 2) such that only a few significantly polarized QSOs are affected (and, as done in Table 4, the most discordant objects may be flagged as contaminated).

This test demonstrates that the distribution of the polarization angles of polarized ( $p \geq 0.6 \%$ ) QSOs is not significantly contaminated by the interstellar polarization, and more particularly after subtracting the systematic correction. But this conclusion is only valid if the interstellar polarization which affects the QSOs is not significantly larger than the polarization measured from field stars.

\subsection{Time variability of the polarization}

For some targets of our sample, several polarimetric measurements do exist. MARK 877, the BAL QSO Q2208-1720, and the binary QSO PKS 1145-071 A\&B have been observed at two epochs (March and May 2002) and do not show any evidence for significant polarization variability. Also, the measurements for MARK 877 and the BAL QSO J2359-12 are in excellent agreement with the values reported by Berriman et al. (1990) and Brotherton et al. (2001), respectively. The four radio-emitters PKS 2203-215, PKS 2204-54, PKS 2240-260 and PKS 1136-13 were previously observed in white light by Fugmann \& Meisenheimer (1988) and Impey \& Tapia (1990). Within the uncertainties and given the different wavelength coverages, we find a good agreement with our polarimetric measurements, i.e. no evidence for a significant variability, including for the highly polarized quasar PKS 2240-260.

On the contrary, the polarization level of PKS 1222+037 grew up to $2.4 \%$ between March and May 2002, indicating that this object might be variable. Our observations also suggest the variability (in both polarization level and angle) of the BL Lac candidate PKS 1216-010 (Londish et al. 2002). Indeed, Visvanathan \& Wills (1998) report for this object a polarization 

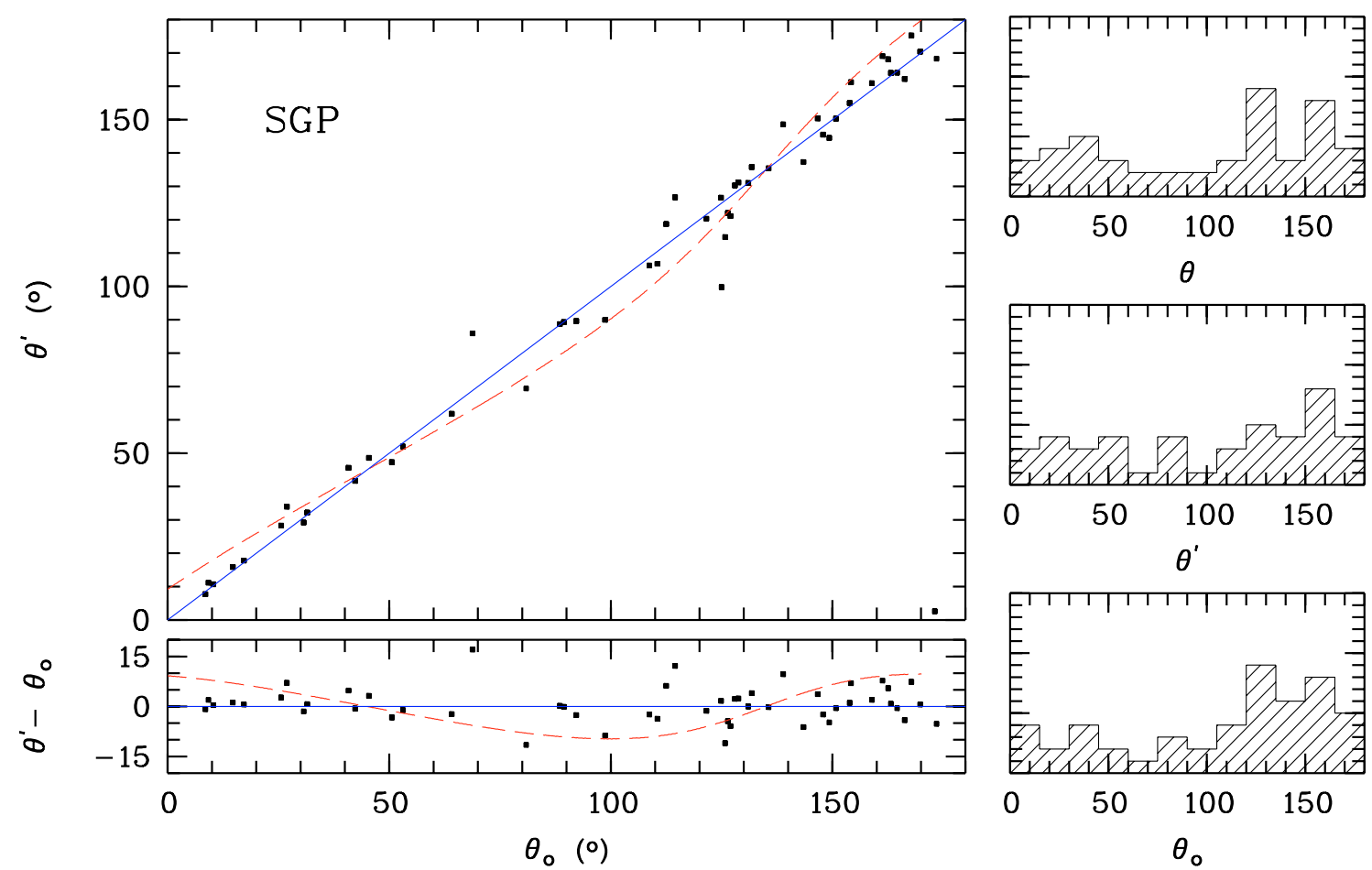

Fig. 5. Same as Fig. 4, but for QSOs from the SGP region (2000 and 2003 data). For most objects, $\left|\theta^{\prime}-\theta_{0}\right|$ is also within $5^{\circ}$. The dashed line represents simulated data obtained by subtracting a systematic polarization of $0.2 \%$ oriented at $135^{\circ}$ (cf. Fig. 1 ) from a polarization of $0.6 \%$ randomly oriented.

level $p=6.9 \pm 0.8 \%$ and a polarization angle $\theta=8 \pm 3.3^{\circ}$ which are significantly different from our values $\left(p_{0}=11.1 \pm 0.19 \%\right.$ and $\theta=100 \pm 0.5^{\circ}$ ). Such a variability is common among highly polarized quasars and BL Lac objects (see e.g. Rieke et al. 1977; Valtaoja et al. 1991).

\section{Conclusions}

New polarization measurements have been obtained for a sample of 203 quasi-stellar objects located in the NGP and the SGP regions with a final median uncertainty $\simeq 0.25 \%$ on the polarization degree. 184 measurements are first time measurements. Among these ones, half of the 42 BAL QSOs show a level of polarization $>1 \%$ and 12 QSOs have a polarization level higher than 3\% (including the BAL QSO SDSS J1409+0048).

Based on previous measurements found in the literature, we find evidence for a variation of both the polarization level and the polarization angle of the BL Lac candidate PKS 1216-010. We also report a significant variability in a two month period of the polarization of PKS $1222+037$. Such a variability is not surprising since this object is reported to have a flat radio spectrum (Teraesranta et al. 2001) which means, in unified schemes, a pole-on view of the AGN with a relativistically beamed polarized emission (Urry \& Padovani 1995; Rusk 1990; Antonucci \& Ulvestad 1985).

The pair of quasi-stellar objects PKS 1145-071 A\&B has been observed at two different epochs. They have polarization levels $>0.6 \%$ and different polarization angles.

This sample also includes the gravitationally lensed QSO J11319-1231 (Sluse et al. 2003). The lensed nature of this object has been serendipitously unveiled on the polarimetric images obtained during the May 2002 observing run. The global polarization level of this object is smaller than $0.3 \%$, confirming that gravitationally lensed QSOs are not more polarized than other quasi-stellar objects (cf. Hutsemékers et al. 1998; and Lam00).

We have also shown that, if the effect of the interstellar polarization in our Galaxy onto distant objects may be adequately represented by the polarization measured from field stars, the significantly polarized ( $p \geq 0.6 \%$ ) QSOs show little contamination in the distribution of their polarization angles.

Acknowledgements. Dominique Sluse acknowledges support from an ESO studentship in Santiago and PRODEX (Gravitational lens studies with HST). Hervé Lamy would like to thank Prof. J. Lemaire and BIRA-IASB for giving him the opportunity to observe in La Silla in March 2002. Hernan Quintana acknowledges partial support from the FONDAP Centro de Astrophysics. This research has made use of the NASA/IPAC Extragalactic Database (NED), which is operated by the Jet Propulsion Laboratory, California Institute of Technology, under contract with the National Aeronautics and Space Administration.

\section{References}

Antonucci, R., \& Ulvestad, J. S. 1985, ApJ, 294, 158

Antonucci, R., Geller, R., Goodrich, R. W., \& Miller, J. S. 1996, ApJ, 472,502

Barkhouse, W. A., \& Hall, P. B. 2001, AJ, 121, 2843

Becker, R. H., White, R. L., Gregg, M. D., et al. 2000, ApJ, 538, 72

Becker, R. H., White, R. L., Gregg, M. D., et al. 2002, ApJS, 135, 227

Berdyugin, A., Piirola, V., \& Teerikorpi, P. 2004, A\&A, 424, 873

Berriman, G., Schmidt, G. D., West, S. C., \& Stockman, H. S. 1990, ApJS, 74, 869 
Brotherton, M. S., Arav, N., Becker, R. H., et al. 2001, ApJ, 546, 134

di Serego Alighieri, S. 1989, in 1st ESO/ST-ECF Data Analysis Workshop, ed. P. J. Grosbøl, et al., 157

di Serego Alighieri, S. 1997, in Instrumentation for Large Telescopes, ed. J. M. Rodriguez Espinosa (Cambridge University Press), 287

Fugmann, W., \& Meisenheimer, K. 1988, A\&AS, 76, 145

Hall, P. B., Anderson, S. F., Strauss, M. A., et al. 2002, ApJS, 141, 267

Heiles, C. 2000, AJ, 119, 923

Hutsemékers, D. 1998, A\&A, 332, 410

Hutsemékers, D., Lamy, H., \& Remy, M. 1998, A\&A, 340, 371

Hutsemékers, D., \& Lamy, H. 2001, A\&A, 367, 381

Impey, C. D., \& Tapia, S. 1990, ApJ, 354, 124

Lamy, H., \& Hutsemékers, D. 1999, The Messenger, 96, 25 (Erratum: The Messenger 97, 23) (Lam99)

Lamy, H., \& Hutsemékers, D. 2000, A\&AS, 142, 451 (Lam00)

Lamy, H., \& Hutsemékers, D. 2004, A\&A, 427, 107

Londish, D., Croom, S. M., Boyle, B. J., et al. 2002, MNRAS, 334, 941

Melnick, J., Dekker, H., \& D’Odorico, S. 1989, EFOSC, ESO operating manual No. 4, Version 2, ESO

Menou, K., Vanden Berk, D. E., Ivezic, Z., et al. 2001, ApJ, 561, 645

Reichard, T. A., Richards, G. T., Schneider, D. P., et al. 2003, AJ, 125, 1711

Rieke, G. H., Lebofsky, M. J., Tapia, S., Kemp, J. C., \& Coyne, G. V. 1977, ApJ, 218, L37
Rusk, R. 1990, JRASC, 84, 199

Schmidt, G. D., \& Hines, D. C. 1999, ApJ, 512, 125

Schmidt, G. D., \& Smith, P. S. 2000, ApJ, 545, 117

Schneider, D. P., Richards, G. T., Fan, X., et al. 2002, AJ, 123, 567

Schneider, D. P., Fan, X., Hall, P. B., et al. 2003, AJ, 126, 2579

Serkowski, K. 1962, in Advances in Astronomy and Astrophysics, ed. Z. Kopal (Academic Press), 1, 289

Serkowski, K., Mathewson, D. L., \& Ford, V. L. 1975, ApJ, 196, 261

Simmons, J. F. L., \& Stewart, B. G. 1985, A\&A, 142, 100

Sluse, D., Surdej, J., Claeskens, J.-F., et al. 2003, A\&A, 406, L43

Smith, P. S., Schmidt, G. D., Hines, D. C., Cutri, R. M., \& Nelson, B. O. 2002, ApJ, 569, 23

Smith, P. S., Schmidt, G. D., Hines, D. C., \& Foltz, C. B. 2003, ApJ, 593,676

Stockman, H. S., Moore, R. L., \& Angel, J. R. P. 1984, ApJ, 279, 485

Teraesranta, H., Urpo, S., Wiren, S., \& Valtonen, M. 2001, A\&A, 368, 431

Turnshek, D. A., Bohlin, R. C., Williamson II, et al. 1990, AJ, 99, 1243

Urry, C. M., \& Padovani, P. 1995, PASP, 107, 803

Valtaoja, L., Sillanpaa, A., Valtaoja, E., et al. 1991, AJ, 101, 78

Véron-Cetty, M.-P., \& Véron, P. 2001, A\&A, 374, 92

Visvanathan, N., \& Wills, B. J. 1998, AJ, 116, 2119

Wardle, J. F. C., \& Kronberg, P. P. 1974, ApJ, 194, 249

Webb, W., Malkan, M., Schmidt, G., \& Impey, C. 1993, ApJ, 419, 494

Weitenbeck, A. J. 1999, Acta Astron., 49, 59 